An inverse problem for the heat equation,JMAA,264,N2,(2001), 691-697. 


\title{
An inverse problem for the heat equation
}

\author{
A.G. Ramm \\ Mathematics Department, Kansas State University, \\ Manhattan, KS 66506-2602, USA \\ ramm@math.ksu.edu
}

\begin{abstract}
Let $u_{t}=u_{x x}-q(x) u, 0 \leq x \leq 1, t>0, u(0, t)=0, u(1, t)=a(t), u(x, 0)=0$, where $a(t)$ is a given function vanishing for $t>T, a(t) \not \equiv 0, \int_{0}^{T} a(t) d t<\infty$. Suppose one measures the flux $u_{x}(0, t):=b_{0}(t)$ for all $t>0$. Does this information determine $q(x)$ uniquely? Do the measurements of the flux $u_{x}(1, t):=b(t)$ give more information about $q(x)$ than $b_{0}(t)$ does?

The above questions are answered in this paper.
\end{abstract}

\section{Introduction}

Consider the heat transfer problem described by the equation

$$
\begin{gathered}
u_{t}=u_{x x}-q(x) u, \quad 0 \leq x \leq 1, \quad t>0, \\
u(x, 0)=0, \\
u(0, t)=0, \quad u(1, t)=a(t),
\end{gathered}
$$

where $a(t)$ is the prescribed temperature, and $q(x)$ is a real-valued integrable function. Assume that $a(t)$ is a pulse-type function, that is,

$$
a(t)=0 \text { for } t>T, \quad \int_{0}^{T} a(t) d t<\infty, a(t) \not \equiv 0 .
$$

In particular, one can choose $a(t)$ to be the delta-function $a(t)=\delta(t)$. Suppose one measures the flux at one of the ends of the rod, either measuring

$$
u_{x}(1, t):=b(t)
$$

or

$$
u_{x}(0, t):=b_{0}(t) .
$$


The questions that are answered in this paper are:

1 ) Does the knowledge of $a(t)$ and $b_{0}(t)$ for all $t>0$ determine $q(x)$ uniquely?

2) Does the knowledge of $a(t)$ and $b(t)$ for all $t>0$ determine $q(x)$ uniquely?

3) How does one calculate $q(x)$ given $a(t)$ and $b(t)$ ?

The answers we give are:

1) The knowledge of $a(t)$ and $b_{0}(t)$ for all $t>0$ does not determine $q(x)$ uniquely, in general. It does, if $q(x)$ is symmetric with respect to the point $x=\frac{1}{2}$, that is, if $q\left(x+\frac{1}{2}\right)=q\left(\frac{1}{2}-x\right)$, or if $q(x)$ is known on the interval $\left[\frac{1}{2}, x\right]$.

2) The knowledge of $a(t)$ and $b(t)$ for all $t>0$ determines $q(x)$ uniquely.

3) An algorithm for computing $q(x)$ given $a(t)$ and $b(t)$ is given.

The answer to question 2) was given in [5] and earlier, under the additional assumption $q(x) \geq 0$, in [1]. The answer to question 1) is new, as far as the author knows. An algorithm for computing $q(x)$ is similar to the one described in [5].

In section 2 the answers to questions 1) and 2) are given, and an answer to question $3)$ is given in section 3 .

\section{Answer to questions 1) and 2).}

Let us Laplace-transform (1.1)-(1.3), (1.5) and (1.6). If $v:=v(x, \lambda):=\int_{0}^{\infty} u(x, t) e^{-\lambda t} d t$, then

$$
\begin{gathered}
v^{\prime \prime}-q(x) v-\lambda v=0, \quad 0 \leq x \leq 1 \\
v(0, \lambda)=0, \quad v(1, \lambda)=A(\lambda), \\
v_{x}(1, \lambda)=B(\lambda), \\
v_{x}(0, \lambda)=B_{0}(\lambda),
\end{gathered}
$$

where $A(\lambda), B(\lambda)$ and $B_{0}(\lambda)$ are the Laplace transforms of $a(t), b(t)$ and $b_{0}(t)$, respectively.

Proposition 2.1. The data $\{A(\lambda), B(\lambda)\}$ known for a set of $\lambda>0$, which has a finite limiting point, determines $q(x)$ uniquely.

Proposition 2.2. The data $\left\{A(\lambda), B_{0}(\lambda)\right\}$, known for all $\lambda>0$, does not determine $q(x)$ uniquely, in general.

If $q(x)$ is known on the interval $\left[\frac{1}{2}, 1\right]$ then $q(x)$ on the interval $\left[0, \frac{1}{2}\right]$ is uniquely determined by the above data.

Also, if $q\left(\frac{1}{2}-x\right)=q\left(\frac{1}{2}+x\right)$, then $q(x)$ is uniquely determined on the interval $[0,1]$ by the above data.

Proof of Proposition 2.1. Let $\varphi(x, \nu)$ solve equation (2.1) with $\lambda=-\nu$ and satisfy the condition

$$
\varphi(0, \nu)=0, \quad \varphi^{\prime}(0, \nu)=1
$$

The solution $\varphi(x, \nu)$ is an entire function of $\nu$ and of $k=\nu^{1 / 2}=i \lambda^{1 / 2}$ (see [4], [6]). 
Since $\varphi$ and $v$ satisfy the first condition (2.2), one has:

$$
v(x, \lambda)=c(\lambda) \varphi(x,-\lambda),
$$

where $c(\lambda)$ does not depend on $x$. Thus

$$
c(\lambda) \varphi(1,-\lambda)=A(\lambda), \quad c(\lambda) \varphi^{\prime}(1,-\lambda)=B(\lambda) .
$$

Note that $v(x, \lambda)$ may be not defined for some $\lambda$, namely for some $\lambda$, namely for $-\lambda=\lambda_{j}$, where $\lambda_{j}$ are the eigenvalues of the problem

$$
\ell \psi_{j}:=-\psi_{j}^{\prime \prime}+q(x) \psi_{j}=\lambda_{j} \psi_{j}, \quad \psi_{j}(0)=\psi_{j}(1)=0 .
$$

Since $\lambda>0$, the condition $\lambda=-\lambda_{j}$ can be satisfies only if $\lambda_{j}<0$. There are at most finitely many negative eigenvalues of the selfadjoint Dirichlet operator $\ell=-\frac{d^{2}}{d x^{2}}+q(x)$ in $H:=L^{2}[0,1]$. For the problem (2.1)-(2.2) to be solvable, when $\lambda=-\lambda_{j}$ it is necessary and sufficient that the appropriate orthogonaltiy conditions are satisfied. Namely one finds

$$
v(x, \lambda)=-\sum_{j=1}^{\infty} \frac{A(\lambda) \psi_{j}^{\prime}(1)}{\lambda+\lambda_{j}} \psi_{j}(x) .
$$

For this series to be defined at $\lambda=-\lambda_{j}>0$ it is necessary and sufficient that $A\left(-\lambda_{j}\right)=0$. Note that $\psi_{j}^{\prime}(1) \neq 0$ by the uniqueness of the solution to the Cauchy problem (see (2.8)).

Since we have assumed $a(t)=0$ for $t>T$, the function $A(\lambda)$ is an entire function of $\lambda$ on the complex $\lambda$-plane. Therefore $v(\lambda)$ is well-defined as a meromorphic function of the parameter $\lambda$ with values in $H$. Note that problem (1.1)-(1.3) is always solvable, but if the operator $\ell$ has negative eigenvalues, then the solution to (1.1)-(1.3) may grow exponentially as $t \rightarrow+\infty$.

From (2.7) one concludes

$$
\frac{B(\lambda)}{A(\lambda)}=\frac{\varphi^{\prime}(1-\lambda)}{\varphi(1,-\lambda)}
$$

since $c(\lambda) \neq 0$. The zeros of the function

$$
\varphi(1, \nu)=0
$$

are precisely the Dirichlet eigenvalues $\lambda_{j}$ of $\ell$, while the zeros of the function

$$
\varphi^{\prime}(1, \nu)=0
$$

are precisely the eigenvalues of the problem

$$
\ell w_{j}=\mu_{j} w_{j}, \quad w_{j}(0)=0, \quad w_{j}^{\prime}(1)=0 .
$$

It is well known (see e.g. [4]) that the knowledge of $\left\{\lambda_{j}\right\}$ and $\left\{\mu_{j}\right\}$ for all $j$ determines $q(x)$ uniquely because two spectra of $\ell$ with the same homogeneous boundary condition 
at $x=0$ and two different homogeneous boundary condition at $x=1$, determine $q(x)$ uniquely.

The zeros of $\frac{B(\lambda)}{A(\lambda)}=\frac{\varphi^{\prime}(1, \lambda)}{\varphi(1, \lambda)}$ are the numbers $\mu_{j}$ and only these numbers, while its poles are the numbers $\lambda_{j}$ and only these numbers.

Proposition 2.1 is proved.

Remark 2.1. A different proof of Proposition 2.1, based on Property $C$ for ODE, is given in [5].

Proof of Proposition 2.2. From (2.6) and (2.4) it follows that

$$
c(\lambda) \varphi(1,-\lambda)=A(\lambda), \quad c(\lambda) \varphi^{\prime}(0,-\lambda)=B_{0}(\lambda) .
$$

Thus

$$
\frac{B_{0}(\lambda)}{A(\lambda)}=\frac{\varphi^{\prime}(0,-\lambda)}{\varphi(1,-\lambda)}=\frac{1}{\varphi(1,-\lambda)}=\frac{1}{\varphi(1, \nu)}, \quad \nu:=-\lambda .
$$

The poles of the function (2.15) are the eigenvalues $\lambda_{j}$, and this is the only information one can get from (2.15).

The knowledge of one spectrum $\left\{\lambda_{j}\right\}$ of $\ell$ determines, roughly speaking, "half of the potential": namely, if $q(x)$ is known on the interval $\left[\frac{1}{2}, \ell\right]$, then the data $\left\{\lambda_{j}\right\}$ known for all $j$ determine $q(x)$ on $\left[0, \frac{1}{2}\right]$ uniquely (see [3], [5], [7]). By the same reason if $q\left(x+\frac{1}{2}\right)=q\left(\frac{1}{2}-x\right)$ then $q(x)$ is uniquely determined on $[0,1]$ by the set $\left\{\lambda_{j}\right\}$ known for all $j$.

Proposition 2.2 is proved.

The information in the data $a(t)$ and $b_{0}(t)$ is equivalent to the information in the ratio $\frac{B_{0}(\lambda)}{A(\lambda)}$. This is especially clear if one takes $a(t)=\delta(t)$ because in this case $A(\lambda)=1$ and $\frac{B_{0}(\lambda)}{A(\lambda)}=\frac{1}{\varphi(1, \nu)}$, so that the information in the ratio is given just by one function $\varphi(1, \nu)$.

Remark 2.2. In [5] and [7] a general uniqueness result is obtained which says that if $q(x)$ is known on $[b, 1], 0<b<1$, where $b$ is an arbitrary fixed number, then the set $\left\{\lambda_{m(j)}\right\}$ determines $q(x)$ on $[0, b]$ uniquely provided that $\sigma \geq 2 b$. Here $\lambda_{m(j)}$ is an arbitrary subset of $\left\{\lambda_{j}\right\}$ such that $m(j)=\frac{j}{\sigma}\left(1+\varepsilon_{j}\right), \sum_{j=1}^{\infty}\left|\varepsilon_{j}\right|<\infty$. So, if $m(j)=j$, then $\sigma=1, \varepsilon_{j}=0, b \leq \frac{1}{2}$. For $b=\frac{1}{2}$ one gets the uniqueness result used in the proof of Proposition 2.2 and obtained in [3]. See also a related result in [2], which implies the result of [3].

Remark 2.3. From our arguments it follows that extra data (1.6) yields, roughly speaking, half of the information that data (1.5) yields, and therefore does not allow one to recover $q(x)$ uniquely. 


\section{$3 \quad$ An algorithm for computing $q(x)$}

If $\{a(t), b(t)\}$ are our data, one takes the Laplace transform and gets $\frac{B(\lambda)}{A(\lambda)}$. One calculates the zeros and poles of this function and gets the numbers $\left\{\lambda_{j}\right\}$ and $\left\{\mu_{j}\right\}$. In the literature (see [4]) there is an algorithm for calculating the spectral function $\rho(\lambda)$ of the operator $\ell$ from the knowledge of $\left\{\lambda_{j}\right\} \cup\left\{\mu_{j}\right\}$. If $\rho(\lambda)$ is found, then the Gelfand-Levitan algorithm allows one to calculate $q(x)$ from $\rho(\lambda)$. This algorithm is described in [4], [5], [6].

In this section we describe an algorithm which is a version of the one described in [6], pp.297-299 (see [5], p. 57), which is quite different from the Gelfand-Levitan one and may be numerically more stable.

Recall that

$$
\varphi(x, \nu)=\varphi_{0}(x, \nu)+\int_{0}^{x} K(x, y) \varphi_{0}(y, \nu) d y, \quad \varphi_{0}(x, \nu):=\frac{\sin (k x)}{k}, \quad k=\sqrt{\nu},
$$

where $K(x, y)$ is the transformation kernel, and

$$
q(x)=2 \frac{d K(x, x)}{d x}
$$

Since $\varphi\left(1, \lambda_{j}\right)=0$, one gets:

$$
\int_{0}^{1} K(1, y) \varphi_{0}\left(y, \lambda_{j}\right) d y=-\varphi_{0}\left(1, \lambda_{j}\right), \quad j=1,2, \ldots
$$

Since the set $\left\{\varphi_{0 j}\right\}:=\left\{\varphi_{0}\left(y, \lambda_{j}\right)\right\}_{\forall j}$, forms a Riesz basis of $H=L^{2}[0,1]$, relations (3.3) allow one to find $K(1, y)$.

Recall that a basis $\left\{h_{j}\right\}$ of a Hilbert space $H$ is called a Riesz basis if there is a linear bounded map $A$ and $A^{-1}$ is a linear bounded operator on $H$, such that $h_{j}=A f_{j}$, where $\left\{f_{j}\right\}$ is an orthonormal basis of $H$ (see [9], p. 148).

Numerically one may look for $K(1, y)$ of the form

$$
K(1, y)=\sum_{j=1}^{J} c_{j} \varphi_{0}\left(y, \lambda_{j}\right)
$$

substitute (3.4) into (3.3) and get a linear system for $c_{j}, 1 \leq j \leq J$. Here $J$ is an arbitrary large positive integer. The matrix of the linear system is the Gram matrix

$$
\left(\varphi_{0 j}, \varphi_{0 m}\right):=\int_{0}^{1} \varphi_{0 j}(x) \overline{\varphi_{0 m}(x)} d x, \quad \varphi_{0 j}(x):=\frac{\sin \left(k_{j} x\right)}{k_{j}}, k_{j}=\sqrt{\lambda_{j}},
$$

which is not ill-conditioned since $\left\{\varphi_{0 j}\right\}$ forms a Riesz basis.

Differentiate (3.1) with respect to $x$ and set $\nu=\mu_{j}$ and $x=1$ to get

$$
0=\varphi_{0}^{\prime}\left(1, \mu_{j}\right)+K(1,1) \varphi_{0}\left(1, \mu_{j}\right)+\int_{0}^{1} K_{x}(1, y) \varphi_{0 j}(y) d y, \quad j=1,2, \ldots
$$


These equations determine uniquely $K_{x}(1, y)$, since $\varphi_{0}^{\prime}\left(1, \mu_{j}\right), K(1,1)$ and $\varphi_{0}\left(1, \mu_{j}\right)$ are known numbers. Thus we can compute $K(1, y)$ and $K_{x}(1, y), 0 \leq y \leq 1$, from the data $\{a(t), b(t)\}$.

If $K(1, t)$ and $K_{x}(1, t)$ are known, then one can derive a Volterra integral equation for the unknown $U:=\{q(x), K(x, y)\}$ (see [5], p.56, and [8]).

In [8] it is proved that this equation can be solved by iterations, and therefore $q(x)$ can be computed by an iterative process.

For convenience of the reader we write down the above integral equation for $U:=$ $\{q(x), K(x, y)\}$ and an iterative process for the solution of this equation:

$$
U=W(U)+h,
$$

where

$$
W(U):=\left(\begin{array}{c}
-2 \int_{x}^{1} q(s) K(s, 2 x-s) d s \\
\frac{1}{2} \int_{D_{x y}} q(s) K(s, t) d s d t
\end{array}\right),
$$

$D_{x y}$ is the region bounded by the straight lines $s=1, t-y=s-x$, and $t-y=x-s$ on the $(s, t)$ plane,

$$
\begin{gathered}
h=\left(\begin{array}{l}
f \\
g
\end{array}\right), \\
f(x):=2\left[K_{y}(1,2 x-1)+K_{x}(1,2 x-1)\right], \\
g(x, y)=\frac{K(1, y+x-1)+K(1, y-x+1)}{2}-\frac{1}{2} \int_{y+x-1}^{y-x+1} K_{s}(1, t) d t .
\end{gathered}
$$

Note that $f$ and $g$ are computable from the data $K(1, x)$ and $K_{x}(1, x)$, and (3.6) is a nonlinear Volterra-type equation for the unknown $q(x)$ and $K(x, y)$.

It is proved in [5], p 57 (and in [8]) that the iterative process

$$
U_{n+1}=W\left(U_{n}\right)+h, \quad U_{0}=h
$$

converges (at a rate of geometric series) to $U(x)=\left(\begin{array}{r}q(x) \\ K(x, y)\end{array}\right)$. The details concerning the functional space in the norm of which the convergence hold are given in [5].

\section{References}

[1] Denisov, A., Introduction to the theory of inverse problems, MGU Press, Moscow, 1994. 
[2] F. Gesztesy and B. Simon, Inverse spectral analysis with partial information on the potential, II. The case of discrete spectrum, Trans. Amer. Math. Soc. 352, 2765-2787 (1999).

[3] Hochstadt, H., Lieberman, B., An inverse Sturm-Liouville problem with mixed data, SIAM J. of Appl. Math., 34, 1976, 676-680.

[4] Levitan, B.M., Inverse Strum-Liouville problems, VNU Press, Utrecht, The Netherlands, 1987.

[5] Ramm, A.G., Property C for ODE and applications to inverse problems, in the book "Operator Theory and Its Applications", Amer. Math. Soc., Fields Institute Communications vol. 25, (2000), pp.15-75, Providence, RI. (editors A.G.Ramm, P.N.Shivakumar, A.V.Strauss).

[6] Ramm, A.G., Multidimensional inverse scattering problems, Longman/Wiley, New York, 1992, pp.1-385.

[7] Ramm, A.G., Property C for ODE and applications to inverse scattering, Zeit. für Angew. Analysis, 18, N2, (1999), 331-348.

[8] Ramm, A.G., Recovery of compactly supported spherically symmetric potentials from the phase shift of s-wave, In the book: Spectral and scattering theory, Plenum publishers, New York, 1998 (ed. A.G.Ramm), pp.111-130.

[9] Ramm, A.G., Scattering by obstacles. Reidel, Dordrecht, 1986, pp.1-442. 